\title{
Ensemble epistasis: thermodynamic origins of non-additivity between mutations Michael Harms ${ }^{1}$, Anneliese Morrison ${ }^{2}$, Daria Wonderlick ${ }^{3}$ \\ ${ }^{1}$ University of Oregon ${ }^{2}$ University of Oregon, ${ }^{3}$ University of Oregon \\ harms@uoregon.edu
}

Macromolecules exist as ensembles of interchanging conformations. As a result, a single mutation to a macromolecule has multiple effects, one on each conformation. These individual effects are then averaged over the ensemble to yield the mutation's overall effect. We recently proposed that this feature of macromolecules could lead to non-additivity between mutations, meaning the effect of one mutation changes the effect of another mutation. We termed this phenomenon "ensemble epistasis." Epistasis is fascinating and important. It greatly increases the difficulty of molecular engineering efforts and hinders predictive models of molecular evolution. I will describe our recent efforts to understand, model, and dissect ensemble epistasis using a combination of experimental and computational approaches. We analytically found the conditions that must be met by a macromolecule for it to exhibit ensemble epistasis. Using structure-based calculations, we found these conditions are likely met by real macromolecules. Based on this work, we predicted we that allosteric macromolecules would exhibit ensemble epistasis with a magnitude dependent on the concentration of allosteric effector. We tested these predictions with experimental measurements of mutant cycles in two molecular switches, the adenine riboswitch (RNA) and the lac repressor (protein). Both exhibited effector-dependent ensemble epistasis with a peak magnitude of up to $2 \mathrm{kcal} / \mathrm{mol}$. This energetic phenomenon is detectable in the in vivo transcriptional output of the lac repressor. We also found that we can discriminate ensemble epistasis from other origins of epistasis (e.g. a direct contacts between mutations). Intriguingly, ensemble epistasis is maximal at the effector concentration corresponding to the off/on midpoint of these molecular switches. The prevalence of ensemble epistasis at these biologically crucial switchover points suggests it plays a profound role in natural molecular evolution. 